\title{
Neural prostheses: clinical applications of functional electrical stimulation in spinal cord injury
}

\author{
P H Peckham PhD, G H Creasey FRCSEd \\ Case Western Reserve University, Veterans Affairs Medical Center, and MetroHealth \\ Medical Center, Cleveland, Ohio, USA.
}

\begin{abstract}
Function lost in spinal cord injury can be partially restored in some patients by electrical stimulation of remaining neurons. Neural prostheses designed for this purpose have been under development for several decades and are now in increasing clinical use. Applications are outlined for restoration of respiration, bladder, bowel and sexual function, exercise, hand grasp and standing and walking.
\end{abstract}

Key words: electric stimulation; paraplegia; quadriplegia; rehabiliation; prostheses.

\section{Introduction}

Electrical stimulation has the potential for exciting virtually every tissue with intact peripheral innervation. Thus it is highly significant for individuals who are disabled with spinal cord injury, since many of their muscles retain lower motor neurons but have an absence of control from higher centers. Functional electrical stimulation (FES) is the use of lower level electrical stimulation to facilitate functional control. FES has had an increasing impact on care and rehabilitation in SCI in several areas, including respiratory control, urinary control, bowel management, sexual function, and control of manipulation and mobility. This paper outlines the state of the art of these applications and the prospects for the near future.

\section{Respiration}

In high tetraplegic patients who do not recover adequate voluntary movement of respiratory muscles, phrenic nerve stimulation can be an alternative to long term

Correspondence: Rehabilitation Engineering Center, Hamann Building, MetroHealth Medical Center, 3395 Scranton Road, Cleveland, Ohio 44109, USA. mechanical ventilation. The lower motor neurons of the phrenic nerve must be intact and the method is thus more suited to patients with tetraplegia at the level of $\mathrm{C} 1$ or $\mathrm{C} 2$ than to those in whom the anterior horn cells at the levels of C3-C5 may be damaged. Assessment of candidates therefore includes testing of the phrenic nerve by stimulation through the skin of the neck while recording the effect on the diaphragm.

A platinum electrode is implanted surgically against the deep surface of the phrenic nerve, either in the neck or by a transthoracic approach through the second intercostal space. Each electrode is connected by a subcutaneous lead to a radio receiver under the skin of the chest. A transmitter outside the body conveys power and control signals to the receivers and thus allows adjustment of respiration.

Glenn et $a l^{1}$ reviewed 477 patients with phrenic pacemaker implants carried out over a period of 20 years. Of this personal series of 77 patients, $52 \%$ were paced for 5 to 10 years and $24 \%$ for 10 to 16 years. ${ }^{2}$ Another multicenter study found that $63 \%$ survived for at least 9 years post injury, and that over $90 \%$ of the high tetraplegics on either phrenic pacing or respirators considered their quality of life to be good or excellent. ${ }^{3}$ Activation of the intercostal 
muscles has been used in a few patients, via either an epidural electrode ${ }^{4}$ or a series of individual electrodes. ${ }^{5}$ This may supplement phrenic pacing when the phrenic nerves are partly damaged.

The phrenic pacemaker has an established place in clinical practice. The number of subjects is small but is growing due to improved emergency services and resuscitation. In view of the severe disability of these patients it is desirable to designate centers and teams to specialize in this form of managament.

\section{Bladder, bowel and sexual function}

Stimulation of the sacral anterior nerve roots can now reduce bladder, bowel and sexual complications of suprasacral spinal cord injury. The sacral parasympathetic fibers must be shown to be intact by urodynamics or by transrectal stimulation. Electrodes are usually placed intradurally on the anterior roots of S2 to S4 via a low lumbar laminectomy, although they can be placed extradurally in the spinal canal if the posterior roots are divided proximally. A posterior rhizotomy is often advised even if the electrodes are intradural. Cables from the electrodes are connected to an implanted radio receiver which receives power and stimuli from a portable transmitter.

Brindley et al reported on the first 50 sacral root implants with a follow up of from 5 to 13 years. ${ }^{6,7}$ The results include substantial reductions in residual volume of urine and in urinary tract infection, and increases in bladder capacity and continence. About $60 \%$ of males can produce penile erection with the device. Other forms of electrical stimulation, via either a rectal electrode ${ }^{8}$ or an implanted hypogastric plexus stimulator, ${ }^{9}$ can produce seminal emission in a significant proportion of spinal cord injured men.

Patients using the sacral anterior root stimulator show an increased frequency of defecation and a reduction in constipation and fecal impaction. ${ }^{10}$ Fifty per cent are able to achieve complete rectal evacuation with the implant without manual help, and the mean time spent defecating each week was reduced in one series from 2.5 hours to less than half an hour. ${ }^{11}$

Over 500 sacral anterior root stimulator implants have been done at 34 centers in 14 countries, and approximately $90 \%$ are in regular use 4 to 6 times a day.

\section{Motor system applications}

Applications of FES to the motor system have focused on 3 primary areas: exercise and muscle conditioning, control of the upper extremities, and control of standing and walking.

\section{Exercise and muscle conditioning}

The use of electrical stimulation for exercise has been primarily focused on reversing muscle atrophy and enhancing cardiovascular fitness. While electrical stimulation has not been effective in maintaining denervated muscle for extended periods, those muscles which have at least partial lower motor neuron innervation can be hypertrophied with chronic electrical stimulation. The result is to increase vascularity and mitochondrial activity to reflect properties that are similar to those of type I (slow) fibers, as well as increase size in the atrophied fibers. These changes have been observed both in controlled animal experi-

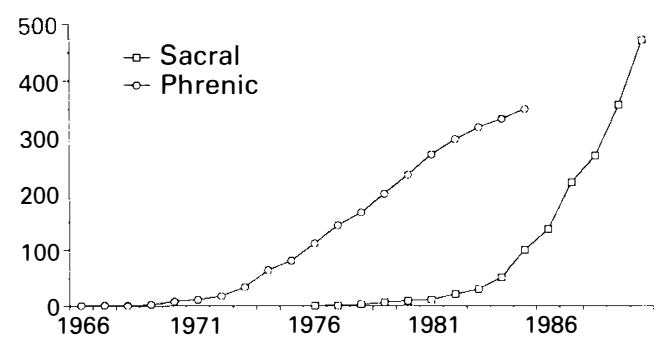

Figure 1 Numbers of phrenic nerve stimulators and sacral anterior root stimulators implanted. Data relates to devices by Glenn and Brindley respectively and has kindly been provided by them. A further 109 patients with phrenic nerve stimulators up to 1986 are not included owing to incomplete follow up. Figures for phrenic nerve stimulator since 1986 have not been obtainable from the manufacturer. 
ments and in humans. Electrical exercise of the lower limbs also increases venous return and systemic fibrinolytic activity. ${ }^{12}$ Electrical stimulation combined with low dose heparin has been shown to be significantly more effective than low dose heparin alone in preventing deep vein thrombosis in acute spinal injured patients. ${ }^{13}$

Cardiovascular capacity is known to deteriorate in spinal cord injury. Electrical stimulation of the lower extremity muscles, generally carried out with cycle ergometry, has shown increased cardiovascular capacity following the implementation of stimulation. ${ }^{14,15}$ As well as the potential health benefits that may result from the adoption of an exercise regime over a lifetime, the results of such a program would be expected to enhance an individual's candidacy for FES standing and walking, which requires strength, endurance, and adequate range of movement in the joints.

\section{Upper extremity control}

Applications of FES to the upper extremities have focused on restoring grasp and release for individuals with C5 and C6 level injuries. ${ }^{16}$ The objective is to reduce reliance on adapted equipment and attendant care and improve independence in performing tasks of daily living. Most activity in this area has used implanted electrodes. Two basic approaches have been taken; chronically indwelling percutaneous electrodes or surgically implanted systems.

In the former, multifilament helically wound electrodes are implanted with a hypodermic needle into the desired muscle, and the needle is withdrawn when the desired response is obtained. Approximately 8 muscles are used to restore both palmar prehension (pinch) and lateral prehension (grasp) and release. Electrodes are implanted via sites located on the dorsal and volar aspects of the distal forearm. This type of electrode has been functional for periods of as long as 5 years, and patients have been continuously implanted and using these electrodes for 13 years. The deficiency in this electrode is that the skin interface needs weekly maintenance, the electrodes are subject to failure, and the electrodes need an external cable that may become entangled. Wijman et l $^{17}$ have shown the increase in function of patients using this system.

The surgically implanted device is similar conceptually to that used for the respiratory or bladder systems, except that 8 channels of stimulation are required. The implantable stimulator-receivers are powered and controlled by external radio signals and provide independent control of each muscle. The external control unit can thus be programmed to synthesize the movement of the muscles to provide smooth grasp. The receiver is implanted on the chest, and the leads are subcutaneously tunnelled to the forearm to terminate on the nerves or muscles. The leads are more substantial than those used percutaneously, and disc electrodes placed on the muscle epimysium have primarily been used. Control of the device is through a proportional joystick, placed on the shoulder or wrist. For the shoulder, protraction and retraction is used to grade opening and closing, and elevation is used to initiate a lock to enable grasp to be maintained. For wrist control, extension controls grasp closure and a switch is used to activate lock. Wrist control has the advantage of augmenting tenodesis pinch. Auditory feedback or sensory feedback is used to provide the user with information regarding the system state (eg on/off, hold/unlock). When sensory feedback is used, an implanted electrode is placed in an area of skin where the patient has normal sensation and the frequency of the stimulus is used to signal the different states.

Primary issues in determining candidacy for the hand system are the range of motion of joints and possible denervation of the forearm and hand muscles. Regarding ROM, patients should not have contractures in the hand which limit extension, because these can restrict the size of objects that can be grasped. Regarding denervation, each individual must be assessed individually. Denervation does not necessarily rule out consideration for FES, as combining FES with tendon transfers of muscles having an upper motor neuron lesion has been found to be useful in substituting for denervated muscle groups. ${ }^{18}$ 
Peckham and Keith ${ }^{19}$ recently performed a survey to determine the number of upper extremity FES systems in use outside the laboratory. Laboratories in Canada, England, Israel, Japan, and the US reported 20 systems in use. There are 4 multichannel implanted systems; 3 in Cleveland and one in London. The longest implanted systems have been functional for over 5 years. Fourteen patients in North America are part of a collaborative study led by the Cleveland team to evaluate the efficacy of percutaneous systems in 5 centers in North America. The study is to determine use and functionality under identical experimental protocols. While completion is not expected until July 1992, the initial results are promising. Each center has successfully implemented at least one user, and all patients report important functional gains. The primary deficiencies noted have been in the laboratory programming interface and in issues associated with percutaneous electrodes (eg skin irritation from tape, cable entanglement). Extension of this work is underway to incorporate the implanted receiver and simplify programming for the therapist.

Extension of this FES application may be expected to benefit individuals with other levels of spinal lesion as well as other disabilities. For example, Nathan ${ }^{20}$ has been investigating elbow control and hand grasp in C4 injury. The Shriner's Hospital of Philadelphia has applied the Cleveland system effectively to a 10 year old with $\mathrm{C} 7$ level injury and is presently extending the hand system for use in cerebral palsy and control of elbow and hand in $\mathrm{C} 4$ level injury.

\section{Lower extremity control}

Applications of FES in the lower extremity have been focused on standing and walking in patients with incomplete and complete lesions at thoracic level. As in the upper extremity, lower level lesions can result in lower motor neuron damage and inability to develop appreciable strength from the denervated muscle. Both surface (cutaneous) and implanted electrodes are being used in research in at least 17 laboratories worldwide. ${ }^{21}$ The goal has generally been to provide reciprocal gait, because of the stability of this gait pattern and its potential energy efficiency. While control is generally achieved by selecting from a menu with the hand, several basic approaches for stimulation have been used. For systems intended to provide standing and minimal walking functions, surface stimulation alone or in combination with minimal orthotic support is the most common approach, as initially recommended by Kralj. ${ }^{22}$ Typically the knee extensors alone or in conjunction with the hip extensors are used. A system of this type is presently undergoing multicenter trials in the United States under the sponsorship of Sygmedics Inc. More advanced walking systems have taken advantage of the flexion withdrawal reflex to evoke hip flexion and ankle dorsiflexion by afferent stimulation, frequently applied to the peroneal nerve. Systems developed in Ljubljana, Slovenia, have added this to the above systems, incorporating either surface or single channel implantable stimulators. Another approach has been to add orthotic support to reduce the number of degrees of freedom that must be provided and give structural support and protection to the limb, which may have osteoporotic bones and asensory joints and skin. Various orthoses have been utilized, including KAFOs which utilize ground reaction forces to help stabilize the limb in stance and HKAFOs which require minimal stimulation to assist in particular motions. Most commonly used in this regard are the reciprocal gait orthoses (RGO) and the hip guidance orthosis (HGO) in combination with surface stimulation of the quadriceps.

One of the principal difficulties associated with the surface stimulation approach is the inability of the electrodes to activate certain deep muscle groups selectively and reliably (eg iliopsoas). Donning of both the stimulating electrodes and the orthosis can also be difficult and time consuming. Implantable systems are under development which are intended to address these issues. Both percutaneous electrodes and implantable stimulator-receivers have been used. The percutaneous electrode provides a versatile approach in enabling the investigator to determine the functional significance pro- 
vided by adding new muscle groups without subjecting the patient to surgical implantation. Marsolais in Cleveland has implanted up to 48 muscles in both legs and the spine using this approach without major complicatiron. ${ }^{23}$ As in the upper extremity, clinical experience in home use is gained with this approach, but improved reliability and long term acceptance will necessitate surgical impantation of the receiver unit. Multichannel implanted receivers have been used in England $^{24}$ and Austria. ${ }^{25}$ Patients were able to demonstrate functional ambulation with these systems in either swing through or reciprocal gait, but none of these systems is operative at present. Several of the units experience infection, which is an important consideration if the volume of implanted material (ie receiver, leads and electrodes) becomes excessive.

Energy consumption with FES walking continues to decrease as the experience in synthesizing movement improves. However, the energy required to walk is still more than twice that of able bodied individuals and greater than that of propelling a wheelchair. The greatest clinical challenge facing this application in SCI is achieving acceptable levels of function and reliability which supplement those functions provided by the wheelchair as a tool for daily mobility.

\section{Clinical systems issues}

Progress toward the use of electrical stimulation to increase the independence and quality of life of SCI patients will present additional clinical challenges in the future. One can conceive of a person with high level quadriplegia who could have implanted systems for control of upper limb function, bladder, and transfer assistance. The implementation of such systems will require interaction with several specialties, and maintenance must be ensured. With considerable use will come breakage of components, and the design and implementation must ensure that these failures can be repaired. Engineering design updates must not necessitate removal of entire systems in the event of failures, but should allow mating of old and new components. Usage must be sufficiently simple that the functions provided are not outweighed by the complexities. The challenge to both engineers and clinicians for the future will be to insure this for the maximal benefit of the patient.

\section{Acknowledgement}

The authors acknowledge the support of the Department of Veterans Affairs Rehabilitation Research and Development Service and the Edison BioTechnology Center.

\section{References}

1 Glenn WWL, Brouillette RT, Dentz B, et al (1988) Fundamental considerations in pacing of the diaphragm for chronic ventilatory insufficiency: a multi-center study. Pacing Clin Electrophysiol 11(2): 2121-2127.

2 Glenn WWL, Phelps ML (1985) Diaphragm pacing by electrical stimulation of the phrenic nerve. Neurosurgery 17(6): 974-984.

3 Whiteneck GC, Carter RE, Charlifue SW (1985) A collaborative study of high quadriplegia. Grant Report No. 133NH50014, National Institute of Handicapped Research, Washington DC.

4 Dimarco AF, Kovvuri S, Petro J, Supinski G (1991) Intercostal muscle pacing in ventilator-dependent quadraplegics. Am Rev Respirat Dis 143: A473.

5 Brindley GS (1991) Personal communication

6 Brindley GS, Polkey CE, Rushton DN, Cardozo L (1986) Sacral anterior root stimulators for bladder control in paraplegia: the first 50 cases. J Neurol Neurosurg Psychiatry 49(10): 1104-1114.

7 Brindley GS, Rushton DN (1990) Long-term follow-up of patients with sacral anterior root stimulator implants. Paraplegia 28(8): 469-475.

8 Bennett AJ, Seager SW, Vasher EA, McGuire EJ (1988) Sexual dysfunction and electroejaculation in men with spinal cord injury: a review. J Urol 139: 453-457.

9 Brindley GS, Sauerwein D, Hendry WF (1989) Hypogastric plexus stimulators for obtaining semen from paraplegic men. Br J Urol 64: 72-77.

10 Binnie NR, Smith AN, Creasey GH, Edmond P (in press) Constipation associated with chronic spinal cord injury: the effect of pelvic parasympathetic stimulation by the Brindley stimulator. Paraplegia.

11 MacDonagh WM, Smallwood R, Forstner D, Reid NW (1990) Control of defecation in patients with spinal injuries by stimulation of sacral anterior nerve roots. Br Med J 300: 1494-97.

12 Katz RT, Green R, Sullivan T, Yarkony G (1987) Functional electric stimulation to enhance systemic fibrinolytic activity in spinal cord injury patients. Arch Phys Med Rehabil 68(7): 423-426. 
13 Merli GJ, Herbison GJ, Ditunno JF, et al (1988) Deep vein thrombosis: prophylaxis in acute spinal cord injured patients. Arch Phys Med Rehabil 69(9): 661-664.

14 Ragnarsson KT, Wolf SL (in press) Physiological considerations and clinical utility of functional electrical stimulation and biofeedback in rehabiliation. In: Downey JA, Myers SJ, Gonzalez E, eds. Physiological Basis of Rehabilitation Medicine. Demos Press, New York.

15 Ragnarsson KT, Pollack S, O’Daniel W, Edgar R, Petrofsky J, Nash MS (1988) Clinical evaluation of computerized functional electrical stimulation after spinal cord injury: a multicenter pilot study. Arch Phys Med Rehabil 69: 672-677.

16 Gorman PH, Peckham PH (in press) Upper extremity functional neuromuscular stimulation. J Neurol Rehabil.

17 Wijman CAC, Stroh KC, Van Doren CL, Thrope GB, Peckham PH, Keith MW (1990) Functional evaluation of quadriplegic patients using a hand neuroprosthesis. Arch Phys Med Rehabil 71: 1053-1057.

18 Keith MW, Peckham PH, Thrope, et al (1989) Implantable functional neuromuscular stimulation in the tetraplegic hand. J Hand Surg 14A(3): 524-530.

19 Peckham PH, Keith MW (in press) Motor prosthesis for restoration of upper extremity function. In: Stein RM, Peckham PH, eds. Neural Prosthesis: Replacing Motor Function after Disease or Disability. Oxford University Press, New York.

20 Nathan RH (1989) Generation of functional arm movements in C4 quadriplegics by neuromuscular stimulation. In: Rose FC, Jones R, Vrbrová G, eds. Neuromuscular Stimulation: Basic Concepts and Clinical Implications. Demos Press, New York: 273-285.

21 Peckham PH (1987) Functional electrical stimulation: current status and future prospects of applications to the neuromuscular systems in spinal cord injury. Paraplegia 25: 274-288.

22 Kralj AR, Bajd T (1989) Functional Electrical Stimulation: Standing and Walking after Spinal Cord Injury. CRC Press, Boca Raton, Florida.

23 Marsolais EB, Kobetic R, Chizeck HJ, Jacobs JL (in press) Orthoses and electrical stimulation for walking in complete paraplegia. J Neurol Rehabil.

24 Brindley GS (1991) MRC Neurological Prosthesis Unit, 1 Windsor Walk, London SE5 8BB, England. (Personal communication.)

25 Thoma H (1991) Ordinariat für biomedizinische Technik u. Physik, A1090 Wien, Sensengasse 8, Austria. (Personal communication.) 